\title{
Malaria Parasite Detection from Microscopic Blood Smear Image: A Literature Survey
}

\author{
Thenu Eliza Thampi \\ Department of Computer Science \\ College of Engineering Poonjar \\ Kerala, India
}

\begin{abstract}
Malaria is a mosquito-borne parasitic disease that is caused by the parasite of the genus Plasmodium. This infectious disease is transmitted via the bite of infected Anopheles mosquitoes. This allows the parasite to enter the human body, and they get matured in the liver and affect the RBC. Malaria is usually found in tropical regions and sub-tropical regions where the climate is suitable for its growth. Every year millions of people are affected by Malaria. In this paper, we focus on the study of different image processing methods for the detection of Malaria infection in humans. A comparison study is made among these methods.
\end{abstract}

\section{General Terms}

Malaria Diagnosis, Image Analysis, Computer Vision, Health Informatics, Machine Learning, Image Processing.

\section{Keywords}

Blood smear image, segmentation, blood cells, classification, parasite detection,

\section{INTRODUCTION}

\subsection{Overview}

Malaria is an infection caused by a few Plasmodium species. They are single-cell species that get spread by mosquitoes. Once Plasmodium enters the blood streams, it infects and destroys mainly the liver cells and blood cells. Even it can cause death. It affects mainly young children under 5 years, pregnant women, patients who have conditions like HIV and travelers with no prior exposure to Malaria. The tropical and sub-tropical regions are affected the most. There are 100s of Plasmodium species but only 5 of them causes Malaria in humans. They are Plasmodium falciparum (P.faliciparum), Plasmodium vivax (P.vivax), Plasmodium ovale (P.ovale), Plasmodium knowlesi(P.knowles)

The infectious disease is transmitted via the bite of infected Anopheles mosquitoes. In their salivary glands, Plasmodium is in a stage of development called sporozoite. Through mosquitoes proboscis the sporozoite spill into the human bloodstreams and within minutes they head to the liver where they engage in asexual reproduction called schizogony. The P.falcium, P.malarial, P.knowlesi undergo asexual reproduction and gets matured in the next 1-2 weeks. The host cells die and sporozite get transformed to merozoites. P.vivax and P.ovale go to dormant. Since they don't divide they are called hypnozoties and may enter a snooze. This period can be called an erythrocytic phase because it takes place outside the RBC. The merozoites get released to blood. They will get bounded to the surface of receptors and invades the RBCs. P. Ovale and P. falciparum invade the RBCs of all ages $P$. vivax invades recticulocytes (young, immature $R B C$ ) .P. malariea and P.knowlesi invade older RBCs. Fig 2(a) and (b)[12] shows infected and uninfected blood cells.

\author{
Sreekumar K. \\ Department of Computer Science \\ College of Engineering Poonjar \\ Kerala, India
}

In the erythrocytic phase of malaria, the merozoites undergo asexual reproduction and series of transformation changes inside the RBC. This phase generally lasts to 2-3 days. Early trophozoites, late trophozoites, schizont, ruptured schizont, merozoites are the different stages of the erythrocytic phase. Some of the merozites undergo gametogony. And the merozoite gets divide and give rise to gametocytes. These gametocytes remain in the RBC and sucked up by female Anopheles mosquitoes from infected humans and undergo further development in mosquitoes gut. They navigate to mosquitoes salivary glands and then to the human body to repeat the cycle. The life cycle of Plasmodium is illustrated in fig 1[11.]. Malaria can affect the lungs, kidney, brain, liver and other organs.

Cycle in Mosquito

Cycle in Human

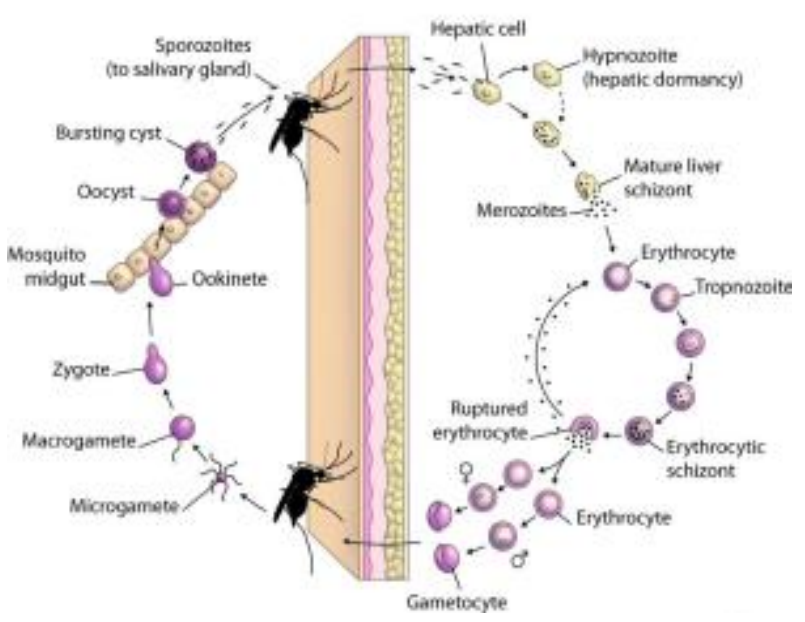

Fig 1: Plasmodium Life Cycle [11]

Microscopic diagnosis is mainly used to detect malarial infections. Thin and thick blood smears in microscopic slides are examined. Thick blood smear helps to identify the malarial infection in RBC and thin blood smear identifies the Plasmodium species. These blood smears are stained with regents like Geimsa for examination under the microscope. But this method is time- consuming and also the result depends on the quality of reagents, microscope and the experience and concentration of the laboratorian.

\subsection{Computer Based Approach}

This work investigates how useful is the computer-aided approach in Malaria parasite detection. It needs digitally captured images of thick and thin blood smears for analysis. The required images of thick and thin blood smears are captured using digital or mobile cameras mounted on the microscope by experts. The data set created in this way includes images of infected and uninfected blood samples, which, then are used as training and testing samples. [12] refers to such a dataset. 


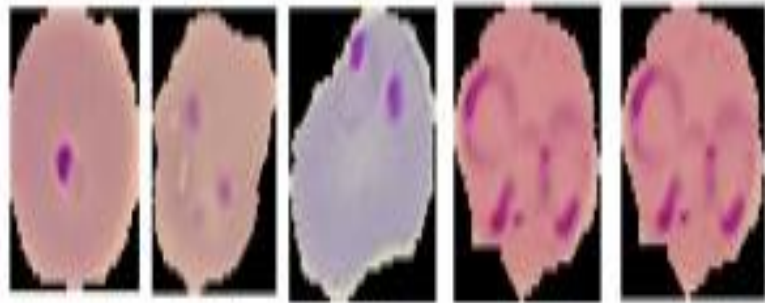

Fig 2(a): Parasitized blood cells [12]

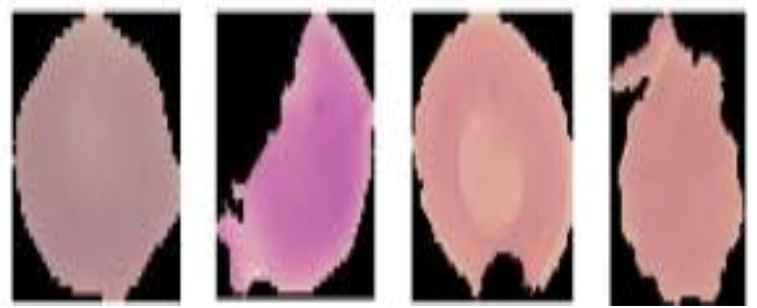

Fig 2(b): Uninfected blood cells [12]

\section{LITERATURE SURVEY}

In 2018, Kunwar, S., Shrestha, M., \& Shikhrakar, R.M.[1.] proposed an image processing method to identify the Plasmodium parasite using the machine learning algorithm .This helps to find out the infected cells and type of parasite based on features.

The image acquisition is the initial step and the database consists of infected and non-infected RBCs images. In order to maintain the $\mathrm{pH}$ level, the images undergo a chemical process which enables the malaria parasite and impurities to be visible. Global thresholding is used for the segmentation of images and creates binary images from gray-scale images. The pixels below a specific threshold is changed to zero and all the pixels above that threshold are changed to one. After this thresholding process the image has undergone enhancement. Erosion and dilation are the morphological methods used here. Watershed segmentation and color segmentation are the two types of segmentation used. Watershed segmentation separates the overlapped RBCs and this separation helps in the counting of RBCs. Color based segmentation helps in defining malaria parasite from impurities. The malaria parasite is larger than the mean value perimeter of RBC cells. The Mathlab function regionprops is used to detect the object perimeter from the image. The parasite perimeter is calculated after finding the mean perimeter of RBC cells. In order to find infected and noninfected cells, parasite perimeter is compared with the mean perimeter of RBC cells. If the mean perimeter of RBC cells is lesser than the perimeter of the parasite, a circle is made around the parasite else nothing is done. After plotting this circle around the parasite the count of infected RBCs is made.

The proposed method was able to find parasite present in the RBCs better than the existing manual method within a small time span.

The study of Kanafiah, S. N. A. M., et al. [2.] introduced a computer-aided diagnosis system that automatically identifies the trophozoite stages of Plasmodium Knowlesi (P.Knowlesi) Malaria, positive and negative control. Image acquisition, processing, and recognition are the different types of image processing methods used here. The Otsu's segmentation the method is used in order to find the region of intersection
(ROI) of infected cells. And recognition using a multilayer perception method is done.

300 thin blood smear images were collected which included 100 images of P.Knowlesi in trophozoite stages, 50 images of gametocyte, 50 images of schizont, 50 images of WBC and 50 images of the artefact. Color medium filter is used to remove salt and pepper noise and it retains the same image data at the same time. Neighboring pixels, nxn has used to yield a better smoothening effect in the color images. Contrast enhancement is applied to images since the infected RBC images captured using a microscope will be a blur. To refine the overall contrast of the image modified global contrast (MGCS) is applied. A weighted sum of RGB components of the image is calculated and then the RGB images are transferred to grayscale images. This conversion is to simplify the images and divided them into two regions. Segmentation based on similarity and dissimilarity is used to extract ROI of infected cells. Feature extraction is done to obtain good accuracy. The sum of the white pixels from the image is used to measure the size of the infected cells and the size of other objects. The multilayer perception is used in the Artificial Neural Network (ANN) classification for recognition. The training classifier identifies trophozoite stages of P.Knowlesi, positive and negative control. Training input is supplied and the coefficient is adjusted to make it suitable for output based on the target within the input features. To train and test for the accuracy of the result Levenberg-marquardt, Bayesian Regulations, Resilient Back-propagation is the algorithms used.

The result shows $90 \%$ of training $10 \%$ of testing accuracy which has $97.67 \%$ correct testing accuracy and the least value of error was $2.33 \%$ compared to other learning algorithms. Multilayer perceptron algorithm of Levenberg-marquardt shows the highest average accuracy. With an accuracy of $98.7 \%$ for training and $97.67 \%$ for testing the recognition of trophozoite stages of P.Knowlesi is done.

An Automatic System for Computing Malaria Parasite Density in Thin Blood Films by Oliveira, Allisson Dantas, et al. [3.] focused in a simple image processing technique that can be implemented to compute the density of malaria parasite in thin blood films. Images are taken using a light microscope with the help of a camera. The pre-processing starts with Gaussian blurring which reduces the noise in the image at a small loss of signal. The output of this process will be used for gray level conversion, whose result is used for the segmentation of the RBC and HSV conversion. Binary image segmentation of RBC is done using intensity channels. The potential plasmodium, platelets and $\mathrm{WBC}$ are detected using HSV converted images. These biological structures acquire more intensive purlish colors when treated with Giemsa. Using sliding window adaptive binarization is performed. For each window the intensities mean and standard deviations (S.D) are calculated. If S.D is smaller than 0.03 then the window is discarded, which implies the absence of RBC in the window .The Otsu approach is used for binarization. Due to the presence lighter nucleus in $\mathrm{RBC}$, the segmentation will produce cells with a hole in their middle which may affect the counting of RBC. And to overcome this situation outer border of RBC is detected and hole filling is performed.by analyzing binary images the connected components are find. The geometrical features such as area, perimeter, compactness of each connected component where find out and these features will help us to find the total number of RBCs in an image. If any connected component does not meet the predefined area and compactness criteria, by performing morphological erosion it is decomposed. This will break the 
components into more. If the criteria for single $\mathrm{RBC}$ are not met by the remainder of connected components, the number of RBCs will be calculated using dividing the area of clusters of cells by 1500 . Using morphological dilation this RBCs are grown again. The parasite density will be valid only if the minimum count of RBC is 500. Using the result of HSV mask in the infected RBCs the RBC, WBC, and Platelets were detected. Centroids of the structures detected on the HSV mask and locate RBCs. If the size of the structure detected on the HSV mask is not defined within a minimum and maximum size, then the plasmodium trophozoites and centroid of the structure are not inside an RBC. And it will be labeled as another element type otherwise it will be marked as an infected RBC cell.

50 images of thin blood films infected with P.falciparum were used as a data set. Classified 20 images as training data set and 30 images as training set and the experiment achieved $89.3 \%$ sensitivity under different illumination and acquisition steps.

In 2018, Abdul-Nasir, Aimi Salihah, et al [4.] introduced a cascaded clustering algorithm for the automatic detection of the malaria parasite. The comparison among the cascaded clustering algorithms is done by considering the accuracy, sensitivity, and specificity of segmented malaria images. Enhanced K-means algorithm (EKM) and fuzzy c-mean (FCM) algorithm were cascaded-means (KM), moving the Kmeans algorithm was cascaded with FCM in order to compare the segmentation performance among this three cascaded algorithms.

The analysis is made on 100 images which were obtained from eight malarial P.vivx slides. Ring, trophozoite, schizont, gametocyte stages were present in the images. The cascaded EKM and FCM clustering algorithms have advantages of both EKM and FCM clustering algorithms. This combines the fundamental theory of both algorithms. So the final center is generated by EKM clustering which will be the initial cluster of FCM clustering. In order to assign members to proper centers during the clustering process the fuzzy membership function is used along with Euclidean distance and variance calculations. FCM algorithm allows assigning of each data to membership grade which indicates the degree of belonging of the data to each center simultaneously using a fuzzy concept. The preprocessing step and it starts by applying the modified global contrast stretching method .It generates images with high contrast .In order to isolate the RBCs and background region the segmentation process, using three cascaded algorithms is done on the green component image. Then the median filter is applied for noise reduction. Seed growing area extraction (SRGAE) is applied to remove unwanted regions like WBC, platelets, artefacts etc. If the segmentation of the malaria parasite is not done then the algorithm will go back to the contrast stretching technique.

A comparison is made between final segmented images and manually segmented images to find the quality of segmentation performance. The cascaded EKM and FCM clustering algorithm obtained the best value compared with other ones.

The Malaria System Microapp: A New, Mobile Device-Based Tool for Malaria Diagnosis by Oliveira, Allisson Dantas, et al [5.] refers to a new mobile device based automated, lowcost system for diagnosis of the Malaria parasite. Image processing and Artificial Intelligent (AI) techniques are used. The face detection algorithm is based on integral images and Haar-like features concepts, weak classifiers with adaptive boosting learning are used. The algorithm helps to identify P.falciparum in the trophozoite stages.

A collection of 500 images of malaria-infected blood slides were used for analysis. In the preprocessing step, the images are converted into gray scale with only single a color space. After this, the gray-scale image was replaced by a single color space, with a black of low intensity and white of higher intensity. For convolution, the Gaussian filter was used. After these operations, the edges of the image and elements of RBCs will become visible. Otsu thresholding is used to separate the gray scale in a given image. After the smoothening of image using the Gaussian filter, it is transformed into binary images containing blood cells only. For the enhancement of geometric structures, the morphological operations erosion is done on the set of pixels. After erosion, a subtraction was applied thus the preprocessing step help in locating in RBC. Training and validation is done to find good classifiers. The images were classified into positive and negative where positive refers to infected cells and negative represent WBC and empty RBC. After this, the adaptive boosting algorithm was applied. A degenerative tree with two negative and positive classes was obtained. An automated mobile device based tool was developed Android phone for the diagnosis of malaria. A second application was also developed to detect parasite in previously acquired images.

The classifier algorithm performed better when a 20-stage cascade was used with average specificity and accuracy values of $96 \%$ and $91 \%$ and respectively.

Bias, Stephen, and Izzet Kale. [6.] Focus on an efficient, easily accessible, networkable, low complexity algorithm for edge detection for the analysis of malaria-infected thin blood smear. Provides an integral solution for the removal of different type of destructive noise, unwanted artefacts and entities. And will make the cell structure suitable for segmentation. The Canny algorithm is used as the baseline.

The dynamic threshold acquisition is calculated using histogram analysis. The algorithm has trained in peak and subsequent peaks will be object peaks. Significant objects are segmented and they may be present atlas $1 \%$ of the total no of pixels. The thresholding surpasses the noise and moves the noise to one side of the boundary or to the other side. The result obtained is a simple binary image that includes all the information's needed. The platelets and other unwanted artefacts have been marked for removal in the binary image. The cells whose centers have intensity value matching with the background in the original gray scale image, and which is not segmented either the platelets are removed by 1) All white components which comprised of 500 or lesser connected pixels are removed. 2) All black components which have comprised of 150 or fewer connected pixels are removed. 3) Any entities tied to cells are removed by morphological closing with small disc-shaped elements that will be in a certain size range and operation can be derived from abovementioned numbers. And this is applied to images of different resolution and zoom. This is combined with thresholding to remove noise effectively. Gray-scale images undergo this process since no significant loss of data in the binary version. They are semi-ambiguous which specifies the edges from binary images and origin pixel in the original image is obtained and marked as an edge. After a number of experiments these rules were refined and the window parts may not be interrogated regardless of their state. The origin with be marked as an edge. This is based entirely on addition and compression that are significantly quicker. 
The algorithm gives high performance and even picks objects from low quality blood images.Hussain, Mahmood, and Dibya JyotiBora [7.] explain about the analytical study of different image segmentation techniques such as thresholding, clustering, histogram-based methods etc.

Thresholding is commonly used for image segmentation. It converts gray-scale image into a binary image. Balanced histogram thresholding and automatic image thresholding are the main methods used. The pixels above a threshold are converted into the foreground and remaining ones to the background of the image. Global thresholding, variable thresholding, and multiple thresholding are the three main types of basic thresholding. Clustering is the segmentation process based on machine learning. For similar images, it applies an indicator. It gives information about a region based on of image set. Mean shift, fuzzy c-mean, K-means are the main clustering methods. For blood image segmentation $\mathrm{K}$ means method is most appropriate and it is widely used for the segmentation of medical images. The histogram based method is more efficient than other methods since it requires only a single pass through image pixels. The image clusters are determined using peaks and valleys of the image. Edge detection is used to find the edge boundaries of pixels. The region-based method can be divided into region growing, split and merge methods. The watershed method uses the concept of gradient magnitude and topological interpretation. Partial differential equation based method is used for time-critical applications. Nonlinear isotropic diffusion filter and covexnon- quadratic variation restoration are the two methods that enhance the edges and remove noise respectively. It helps in noise reduction and better detection of boundaries and edges. And the result shifts the boundaries and edges using close operator. The ANN is used medical image segmentation process. It is helpful in separations ROI from the background. It has a large number of connected nodes and each node has weight associated with it.

Mustafa,W. A., and Aimi Salihah \& Mohamed, Z. (2018) [8.] employed on a new method based on the modification of the Sauvola algorithm. The objective was to improve efficiency in the segmentation for the detection malaria parasite in P. Vivid species and a comparison is made with local segmentation approaches such as local adaptive, Feng, Bradley, Sauvot and Nicks methods. Sauvola method has the ability to solve the problem of black noise. But it cannot segment images if the contrast between the foreground and the background image is small. The proposed method is to overcome this drawback by obtaining the maximum threshold. In order to improve segmentation degraded and noisy sample images are modification using the Sauvola algorithm and it is done by binarization of threshold. This method increases the threshold value and ignorer to segment the information in a low contrast region and also for high and artefact on the resulting images. The maximum and specific threshold values are proposed and ignores to replace the original mean maximum mean was calculated. Using this algorithm low contrast problem can be solved and binarization result is increased automatically 50 malaria-infected images were tested using different segmentation method and compression was made. The 50 sample malaria images were tested. In order to compare the best contrast, the images are separated into RBC channels. Based on observation green channel was selected as the input image for the segmentation process since the target region is highlighted most compared to other channels. And image undergoes segmentation based on different methods. In order to evaluate the performance of segmentation techniques accuracy, sensitivity and specificity were calculated. Based on the comparison of the pixel similarity of resultant images with manual images quality of image segmentation was determined. The Sauvola method achieved the highest result in terms of the sensitivity of $91.09 \%$.

The proposed method shows the most efficient and effective result with a specificity of $99.4 \%$ and accuracy than other methods.

Rajaraman, Sivaramakrishnan, et al [9.] analyzed the performance of pre-trained CNN based DL model's towards feature extraction for the classification of parasitized and uninfected cells for improved disease detection. Other important contributions are 1)the presentation of a comparative analysis of performance of customized and pretrained DL models as feature extractors towards classifying parasitized and uninfected cell 2)cross-validating the predictive models performance at patient level to reduce bias and generalization errors. 3) Analysis and selection of the primal layer in the pre-trained models to extract features from the underlying data. 4) Testing for the presence of the statically significant difference in the performance of customized and pre-trained CNN models under study. Images of Giemsa-stained thin blood smears slides are taken using a mobile application that runs on standard Android segment RBC.The Level set based algorithm is used. 27,558 cell images are present in the dataset with equal instances of infected and uninfected cells. The predictive models are evaluated through five-cross validation which is performed at a patient level to ensure alleviating model biasing and error generalization. The images were resampled and made suitable to customized and pre-trained CNNs. And for faster convergence it is normalized. The models were trained and tested on different CPU's, graphical processing units. The customized, sequential CNN's performance was evaluated in the task classifying parasitized and uninfected cells. And also propose a sequential $\mathrm{CNN}$ for image classification. It has 3 convolutional layers and two fully connected layers. The input to this architecture constitutes segmented cells of $100 \times 100 \times 3$ pixel resolution strides. The first and second convolution layers contain 32 filters and the third has 64 filters. The convolutional layer is followed by a maximum pooling window and 2 -pixel strides. Max pooling layer summaries the output of neighboring neurons groups in feature maps. The output of third convolutional layer is sent to the first fully connected layer that has 64 neutrons and a second fully connected layer feeds into the Softmax classifier. With a dropout ratio of 5 dropout regularization is applied to the output of the first fully connected layers. The model is trained using the optimization of multinomial logistic regression objective using stochastic gradient descent and nester of momentum. The customized model is optimized. In terms of accuracy, the performance of the customized model is evaluated. Performance of pre-trained $\mathrm{CNN}$ is evaluated including Alex Net VGG-16 exception, ResNet-50, and Denset Net-121. Towards extracting the features from infected and uninfected cells. The optimization is done on the model for hyperparameters by the randomized grid search method initialized the search ranges [1e-5- 5e-2], [0.8 0.99 ] and $[1 \mathrm{e}-101 \mathrm{e}-2]$ to find the learning rate. The convolution part of the pertained $\mathrm{CNN}$ was instantiated with dropout. Determines an optimal layer for the feature extraction for the improved classification process. The performance of retrained CNN was evaluated in terms of accuracy, sensitivity, specificity, F1 score and MCC. Statically analysis was performed to choose the best modified for deployment. One way analysis of variance is the method used determines the presence or absence of a significant difference between the 
mean of 3 or more individual unrelated groups. Nonparametric alternatives where used were condition is violated a post-hoc analysis will be needed to demonstrate a specific group with a statically significant difference.

Cell segmentation and detection level-set based algorithms is applied to detect and segment RBC cell detection were done by the application of multi-scale Laplacian of Gaussian filter to detect the centroids of individual RBC. The cell segmentation is done using the generated markers. To remove false detection objects morphological opening operation is applied as post-processing. One to one correspondence is used to remove WBC. Manual pointwise annotation is used to detect the cells using one to one point matching scheme. In terms of accuracy there was significant difference in performance between differences CNN's. The post-hoc test should statistically significant difference existed between retrained Xception, VGG-16, and ResNet-50 and customized model. In the performance between Exceptions, ResNet-50, VGG16, and DenseNet-121 statically significant differences between models in terms of sensitivity and specificity wereobserved. RestNet-50 gained the highest mean rank in terms of accuracy and specificity F1 score and MCC.

The model converged to an optimal solution due to the hyperparameter optimization, implicit regularization imposed by smaller convolution filter sizes and aggressive dropout in the fully connected layers.

Bibin, Dhanya, Madhu S. Nair, and P. Punitha [10.] proposed a method to identify the presence of malaria parasite in human blood smear using deep belief networks (DBN). Introduce a DBN based trained model to classify the images to the parasite and non-parasite class. Restricted Boltzmann machines are used to the pre-train the proposed DBN. Features were extracted for pre-training of DBN. Color and texture features are concatenated are used for feature vector. And the DBN is fine-tuned using Backpropagation algorithm. The Level set method is used for the extraction of stained objects from peripheral blood smears. Level set methods are ideal for segmentation of complex images. Uses concatenated features of color and texture to train the DBN color histogram, color coherence vector (CCV), gray level co-occurrence matrix (GICM). Texture features based on grey level run length matrix (GLRLM) local binary feature (LBD) are the feature extraction methods used. From the experiment, it showed that the no of hidden layers changes in the DBN architecture, the result also changes. And the study also made by varying the size of hidden nodes while having a fixed no of layers.

Provided a unique deep learning based binary classifier that provides significantly higher sensitivity and specificity.

Table 1. Comparative Evaluation of Approaches

\begin{tabular}{|c|c|}
\hline Paper & Remarks \\
\hline [1.] Kunwar, S., & $\begin{array}{c}\text { Propose a method that combines } \\
\text { image processing and machine } \\
\text { Shrestha, M., \& } \\
\text { learning methods for the detection of } \\
\text { the Malaria parasite within a short } \\
\text { span of time. }\end{array}$ \\
\hline $\begin{array}{c}\text { [2.] Kanafiah, S. } \\
\text { N. A. M., et al }\end{array}$ & $\begin{array}{c}\text { Introduced a computer aided system } \\
\text { for the detection of the Malaria } \\
\text { parasite automatically. }\end{array}$ \\
\hline
\end{tabular}

\begin{tabular}{|c|c|}
\hline $\begin{array}{l}\text { [3.] Oliveira, } \\
\text { Allisson Dantas, } \\
\text { et al. }\end{array}$ & $\begin{array}{l}\text { Employed on a simple image } \\
\text { processing technique to detect } \\
\text { parasite density from this blood } \\
\text { smear images. Suitable for images } \\
\text { which are taken at different } \\
\text { acquisition and illumination. }\end{array}$ \\
\hline $\begin{array}{l}\text { [4.] Abdul-Nasir, } \\
\text { Aimi Salihah, et } \\
\text { al. }\end{array}$ & $\begin{array}{l}\text { Focus on a cascaded clustering } \\
\text { algorithm for the automatic } \\
\text { detection of malarial infection. And } \\
\text { a comparison is made between the } \\
\text { proposed algorithm and few } \\
\text { cascaded algorithms. }\end{array}$ \\
\hline $\begin{array}{l}\text { [5.] Oliveira, } \\
\text { Allisson Dantas, } \\
\quad \text { et al. }\end{array}$ & $\begin{array}{l}\text { Proposed an approach based on a } \\
\text { mobile based automated system for } \\
\text { the detection of malarial parasite } \\
\text { using image processing and } \\
\text { Artificial Intelligence. }\end{array}$ \\
\hline $\begin{array}{l}\text { [6.] Bias, Stephen, } \\
\text { and Izzet Kale. }\end{array}$ & $\begin{array}{l}\text { Introduced a mobile based dynamic } \\
\text { system for the detection of malarial } \\
\text { parasite using edge detection } \\
\text { techniques. }\end{array}$ \\
\hline $\begin{array}{l}\text { [7.] Hussain, } \\
\text { Mahmood, and } \\
\text { Dibya JyotiBora. }\end{array}$ & $\begin{array}{c}\text { Study on different image processing } \\
\text { technique in detail for the detection } \\
\text { of Malaria parasite. }\end{array}$ \\
\hline $\begin{array}{l}\text { [8.] Mustafa, W. } \\
\text { A., Aimi Salihah } \\
\text { Abdul-Nasir, and } \\
\text { Zeehaida } \\
\text { Mohamed. }\end{array}$ & $\begin{array}{l}\text { Propose an efficient method for the } \\
\text { segmentation of malaria parasite } \\
\text { using modified Sauvola algorithm. }\end{array}$ \\
\hline $\begin{array}{l}\text { [9.] Rajaraman, } \\
\text { Sivaramakrishnan, } \\
\text { et al. }\end{array}$ & $\begin{array}{l}\text { Made study on a pre-trained CNN } \\
\text { based DL model for the feature } \\
\text { extraction for the efficient detection } \\
\text { of the malaria parasite. }\end{array}$ \\
\hline $\begin{array}{l}\text { [10.] Bibin, } \\
\text { Dhanya, Madhu } \\
\text { S. Nair, and P. } \\
\text { Punitha. }\end{array}$ & $\begin{array}{l}\text { Developed a Deep Learning method } \\
\text { for the detection of malarial parasite } \\
\text { using the Deep Belief Network. }\end{array}$ \\
\hline
\end{tabular}

\section{CONCLUSION}

Malaria is life-threatening diseases that affect millions of people in this world. Different types of automatic systems have been developed for identifying the presence of the Malaria parasite in human blood. We have conducted a comprehensive study of image processing methods to detect the presence of Malaria parasites in the Blood smear. Most of the authors have proposed machine learning approaches after the preprocessing and segmentation steps. Innovations can be suggested in improving the segmentation accuracy as well as in improving detection accuracy. Many attempts have been made with deep learning as well and remarkable accuracy has been reported. The future direction may be in integrating a successful model into a decision support system.

\section{REFERENCES}

[1] Kunwar, S., Shrestha, M., \& Shikhrakar, R.M. (2018). Malaria Detection Using Image Processing and Machine Learning. CoRR, abs/1801.10031.

[2] Kanafiah, S. N. A. M., et al. "An Intelligent Recognition Procedure for Trophozoite Stages of Plasmodium Knowlesi Malaria." Journal of Telecommunication, 
Electronic and Computer Engineering (JTEC) 10.1-16 (2018): 31-35.

[3] Oliveira, Allisson Dantas, et al. "An automatic system for computing malaria parasite density in thin blood films." Iberoamerican Congress on Pattern Recognition. Springer, Cham, 2017.

[4] Abdul-Nasir, Aimi Salihah, et al. "The cascaded moving $\mathrm{k}$-means and fuzzy c-means clustering algorithms for unsupervised segmentation of malaria images." AIP Conference Proceedings. Vol. 1660. No. 1. AIP Publishing, 2015.

[5] Oliveira, Allisson Dantas, et al. "The malaria system microApp: a new, mobile device-based tool for malaria diagnosis." JMIR research protocols 6.4 (2017).

[6] Bias, Stephen, and Izzet Kale. "Mobile Hardware Based Implementation of a Novel, Efficient, Fuzzy Logic Inspired Edge Detection Technique for Analysis of Malaria Infected Microscopic Thin Blood Images." Procedia Computer Science 141 (2018): 374-381.

[7] Hussain, Mahmood, and Dibya JyotiBora. "An Analytical Study on Different Image Segmentation Techniques for Malaria Parasite Detection." 2018 International Conference on Research in Intelligent and Computing in Engineering (RICE). IEEE, 2018.
[8] Mustafa, Wan \& Abdul Nasir, Aimi Salihah \& Mohamed, Z. (2018). Malaria parasites segmentation based on sauvola algorithm modification. Malaysian Applied Biology. 47. 71-76.

[9] Rajaraman, Sivaramakrishnan, et al. "Pre-trained convolutional neural networks as feature extractors toward improved malaria parasite detection in thin blood smear images." PeerJ 6 (2018): e4568.

[10] Bibin, Dhanya, Madhu S. Nair, and P. Punitha. "Malaria parasite detection from peripheral blood smear images using deep belief networks." IEEE Access 5 (2017): 9099-9108.

[11] "Life cycle of the malaria parasite" from Epidemiology of Infectious Diseases. Available at http://ocw.jhsph.edu. Copyright (C) Johns Hopkins Bloomberg School of Public Health. Creative Commons BY-NC-SA.

[12] Rajaraman S, Antani SK, Poostchi M, Silamut K, Hossain MA, Maude, RJ, Jaeger S, Thoma GR. (2018) Pre-trained convolutional neural networks as feature extractors toward improved Malaria parasite detection in thin blood smear images. PeerJ6:e4568. 